\title{
FORMATION OF SPEECH SKILLS IN ONLINE TEACHING RUSSIAN AS A FOREIGN LANGUAGE
}

\author{
Olga A. Petrova ${ }^{1}$, Alena Yu. Nikitina ${ }^{2 \star}$, Tatyana N. Romanova ${ }^{3}$, Nadezhda A. \\ Fedorova $^{4}$, Anzhelika G. Abramova ${ }^{5}$, Elena A. Ignatieva ${ }^{6}$ \\ ${ }^{1}$ Assoc. Prof., Chuvash State University, RUSSIA, zadumka@mail.ru \\ ${ }^{2}$ Assoc. Prof., Chuvash State University, RUSSIA, alyona.nikitina@gmail.com \\ ${ }^{3}$ Assoc. Prof., Chuvash State University, RUSSIA, romanovatn1@mail.ru \\ ${ }^{4}$ Assoc. Prof., Chuvash State University, RUSSIA, fedorovana62@yandex.ru \\ ${ }^{5}$ Assoc. Prof., Chuvash State University, RUSSIA, foreign-languages-department@mail.ru \\ ${ }^{6}$ Assoc. Prof., Chuvash State University, RUSSIA, dima2010ivanoff@mail.ru \\ ${ }^{*}$ Corresponding Author
}

\begin{abstract}
From the point of view of the current state of the methodology of teaching a foreign language, mastering a foreign language occurs if the foreign speaker has developed communicative competence, the ability to solve communicative problems by speech means is formed. Even though the presence of linguistic and speech competence is considered as an intermediate link, the success of communication largely depends on the degree of formation of speech skills, their automation, which is developed by playing many situations using the appropriate speech models (Passov, 2010). During a pandemic, sometimes the only possible form of distance learning and the absence of a language environment become conditions that are fundamentally different from the conditions of communication, which further complicates the teacher's task to form the student's speech skills capable of transferring to the situation of real communication. In addition, the factors of repeatability of speech samples, the regularity of their presentation, so necessary for the automation of speech skills, can cause boredom in the student and even the desire to stop learning a new language for him. Thus, in our opinion, in a distant format, it becomes especially important for a teacher to make the process of teaching speech models at the initial stage of language learning as interesting, variable, and effective for memorization as possible. The aim of the study is to describe the most effective approaches to the formation of speech skills among foreign students, considering the disadvantages of online teaching of Russian as a foreign language.
\end{abstract}

Keywords: online learning, Russian as a foreign language, teaching foreign languages, organizing an online lesson, the mechanism of involuntary memorization, speech skill

\section{INTRODUCTION}

The sudden onset of the Covid-19 pandemic has significantly changed the concept of language education, considering the prevailing new realities. Learning a foreign language from a native speaker via the Internet has become the main mean of creating a natural language environment. Through such training, a dialogue of cultures, intercultural communication takes place. For students who find themselves outside the country of the target language, the teacher on the other side of the screen is a source of knowledge about the linguistic and sociolinguistic features of the target language, the culture of another people, which helps to realize the importance of cultivating tolerance to this culture in oneself, to find a way to mutual understanding. The 
organization of such communication, colored with emotionality, trust in relationships and the authority of the teacher, is currently possible on various educational paid / free platforms, messengers: Zoom, My Webinar, Microsoft Teams, Google Meets, WhatsApp, Viber, Skype, Telegram, and others, which are synchronous online learning is combined with the use of social networks, interactive tutorials, electronic libraries, as well as traditional paper versions of books and dictionaries.

At the same time, questionnaires, various types of surveys related to identifying the attitude of students to the distance learning process, record technical and organizational deficiencies that arise when using the online learning model: unstable and sometimes weak Internet connection; working with foreign listeners who turned off their cameras; psychological difficulties, lack of personal contact; lack of control over turning on / off the microphone from the side of the listener; the presence of distractions at home; difficulty checking homework insufficient skills of working in an online format, as well as imperfect technology of material presentation in general, imperfect forms of control, a small number of interactive textbooks and teaching aids - of course, all this reduces the productivity and efficiency of teaching a foreign language.

Considering the above, teachers, first, are recommended to carefully prepare and think over the organization of each online lesson as much as possible, which will certainly lead to pedagogical success and determine the formation of a stable speech skill of the student. And if technical problems, which are less dependent on the teacher, are gradually being solved, then the organizational difficulties that the teacher encounters arise regularly and require his attention and timely and sometimes non-standard solutions.

The aim of the undertaken research is to describe the most effective approaches to the formation of speech skills of foreign students, considering the disadvantages of online teaching of Russian as a foreign language.

\section{RESULTS}

Studies in the field of teaching a second foreign language have confirmed that the factor of repeatability of speech models in the formation of the automation of a speech skill can cause boredom in the student and, as a result, reduce the motivation for mastering a foreign language until the termination of its study (Kruk, Pawlak, Zawodniak, 2021). The disadvantages of online learning listed above can only lead to an intensification of the decrease in the student's motivation. Understanding this problem forces teachers in the existing conditions of a distance student to look for new solutions that contribute to the activation of the student's mechanism of involuntary memorization of speech models and the creation of conditions that are as close as possible to the conditions of real communication. For this purpose, already at the initial level, interactive tutorials are used, offering tasks with ready-made graphic templates for filling out a business card, greeting card, participating in correspondence in a mobile phone messenger, audio files of recorded dialogues against the background of the corresponding noise situation (in the city, office, airport, restaurant, etc.). For the same purpose, it is proposed to use templates of virtual backgrounds for video conferencing on various platforms to create an atmosphere of an educational situation from business to informal and friendly, depending on the speech task (in the office, in a conference room, in the classroom, at work, at the blackboard, in a cafe, at a football match, at a yoga class, on vacation by the sea, in an uncleaned room, etc.).

It is known that making eye contact with a trainee is an important condition for productive learning. At the same time, the reluctance of many foreigners to turn on the webcam when studying Russian as a foreign language online requires additional efforts on the part of the teacher to ensure the learning process in such a way that such contact between the teacher and the students is certainly established. It is noticed that the inclusion of tasks in the learning process, which, on the one hand, provide visual contact of students with each other and students with the teacher of tasks, and, on the other hand, involve the human motor systems, promotes faster memorization of speech models. In addition, visual contact is a natural condition for real situations of oral communication, practiced at the initial level of teaching a foreign language. Obviously, with the screens turned off, situations that are as close to real as possible are much more difficult to simulate.

To teach foreigners to turn on cameras in the classroom, from the very beginning, you can give tasks for teaching speech models, focusing the attention of students on the intonation of their pronunciation. It is proposed to actively use the student's hand, the movement of which, when pronouncing models in accordance with the necessary intonation structure, is followed by the teacher and the student: for example, when pronouncing a sentence in accordance with IC-1 (affirmative sentence), the palm of the hand moves from the position in front of him to the position down, and vice versa, when pronouncing an interrogative sentence (IC-3), the palm of the hand from a position in front of you moves to a position up: This is a table. Is this a table? Tasks with physical activity controlled by the teacher and students can also be used when using constructions with adverbs with spatial meaning (there, here, here, next to, above, below, left, right, opposite, behind, etc.). 
Ensuring an inextricable connection between all types of activities and their more frequent, in comparison with contact classes, turnover in teaching Russian as a foreign language in an online format is a prerequisite for the development of speech skills and speech skills. The recommended time for completing tasks within one type of speech activity is no more than 20 minutes, and it has been noticed that the assimilation of speech models is more effective not only when changing types of speech activity, but even simply when changing the displayed textbook / study guide, since the change in color and graphic solving the background of the educational material, fonts, the inclusion of different types of illustrations also activate the student's attention and contribute to the memorization of speech models.

According to recent research in cognitive psychology, the mere presence of other people in a visual scene mainly shapes how observers understand the scene, prompting observers to pay more attention to the abstract relationships between objects and people, rather than just the specific features of the objects themselves. Humans have been shown to be more likely to process visual scenes in terms of the underlying structure relationships when a different person is involved in the scenes, not just non-human objects. These results reflect the tendency towards personality-oriented interpretations in understanding the external world, in which the unique and distinctive features of various objects are constructed in the context of their relationships with people (Kalkstein, Hackel, Trope, 2020). Considering the results of these studies, when working out speech models in dialogical and monologue speech, it is necessary to involve students in the process of teaching a foreign language using anthropocentric illustrative material, to a greater extent motivating and stimulating the student to speak, build statements about a person in his interaction with surrounding objects (Nikitina, Petrova, 2019, p. 217).

The reliance on the mechanisms of involuntary memorization of speech models is especially relevant when teaching Russian as a foreign language online, therefore, the use of all kinds of game applications for learning foreign languages, chat bots, including linguistic, visual, auditory presentation, is a particularly highly productive means for memorizing phrases and expressions speech model and small tasks that allow you to control the assimilation of the material - tests with a choice of answer.

In addition, it seems advisable to periodically introduce tasks for students to work in pairs and small groups, for example, playing out situational dialogues, retelling small texts, etc., which are accompanied by control, assessment of each other in accordance with the scales prepared in advance by the teacher, followed by analysis and analysis of the admitted ones mistakes. The data of this type of control, along with information obtained as a result of formal and informal control, the teacher will be able to use for individual consultations and as an additional source for drawing up a general picture of the mastery of the material by the student, studying his needs, adjusting goals and other components of the language teaching system, which is an important and necessary component in the effective work of a teacher when teaching in an online format (Polyakov, 2012).

\section{CONCLUSIONS}

Methods, campaigns, and assignments for online teaching Russian as a foreign language, for sure, will change and improve, but unpredictability, the ability to surprise students with the novelty of the content and form of an online lesson should be its mandatory characteristics, significantly affecting the intensification of the development and consolidation of speech skills and skills.

\section{REFERENCE LIST}

Kalkstein, D. A., Hackel L.M., Trope Y. (2020). Person-Centered Cognition: The Presence of People in a Visual Scene Promotes Relational. Journal of Experimantal Social Psychology, volume 90.

Kovaleva, E. G. (2021). Distance learning Russian as a foreign language: Necessary and sufficient (based on survey data of university students and Russian as a foreign language courses). Russian as a foreign language in a multicultural space: Traditional and digital technologies.

Kruk, M., Pawlak, M., Zawodniak, J. (2021). Another look at boredom in language instruction: The role of the predictable and the unexpected. Studies in second language learning and teaching, volume 11, (issue 1).

Nikitina, A. Yu., Petrova, O. A. (2019). Teaching Russian speech on the Russian as a foreign language lessons. Development of higher education export capacity: content, experience, prospects.

Passov, E. I. (2010). Fundamentals of communication theory and technology of foreign language education: 
Proceedings of INTCESS 2022- 9th International Conference on Education \& Education of Social Sciences 17-18 January 2022- Online Conference

a methodological guide for teachers of Russian as a foreign language.

Polyakov, O. G. (2012). Control in teaching foreign languages. Methods of teaching foreign languages: traditions and modernity. 